\title{
Overview of Electric Vehicle Machines - From Tesla to Tesla, and Beyond
}

\author{
K.T. Chau \\ Department of Electrical and Electronic Engineering, The University of Hong Kong (ktchau@eee.hku.hk)
}

Topics: EM2-Electric Machines Applications

\begin{abstract}
Starting from the rotating magnetic field theory developed by Nikola Tesla in 1882, the induction machine has become the most popular electric machine for over a century. Actually, the Tesla's AC Induction Motor is dubbed one of the ten greatest discoveries of all time. This popularity has been extended to the latest Tesla Motors which has set a new world speed record for production electric vehicles (EVs). Meanwhile, the permanent-magnet (PM) brushless machines are superseding the induction machine because of their high efficiency and high power density. In order to fulfill the ever increasing demand on electric propulsion, innovation of electric machines accelerates. For instance, the stator-PM machines combine the merits of both PM brushless and switched-reluctance machines to offer energy-efficient robust operation; the vernier PM in-wheel machines incorporate the vernier effect into the PM brushless machines to provide low-speed high-torque direct-drive operation; and the advanced magnetless machines adopt innovative topologies to compensate the reduction of torque and power densities due to the absence of PM material. Increasingly, the development of electric machines has been expanded to incorporate the heat engine for hybrid propulsion. Rather than using the planetarygeared machine system to perform power split for electronic continuously variable transmission (ECVT), the double-rotor machine system is developed to achieve gearless ECVT, and the magneticgeared machine system is proposed to achieve ECVT without physical gearing or brushes. In this paper, various electric machines and machine systems for electric and hybrid vehicles will be discussed, with emphasis on the challenges and opportunities of those emerging machine topologies.
\end{abstract}

\section{EV MACHINE REQUIREMENTS}

Electric machines are one of the core technologies for EVs and hybrid EVs (HEVs), which convert the limited on-board electrical energy to the desired vehicular mechanical motion. The corresponding requirements are much more stringent than that for industrial applications [1],[2]:

- High torque and power densities.

- High torque capability for electric launching and hill climbing.

- High efficiency over wide torque and speed ranges.

- Wide-speed operating range for low-speed creeping and high-speed cruising.

- Wide constant-power operating capability.

- High intermittent overload capability for overtaking.

- High reliability and robustness for vehicular environment.

- Publicly acceptable cost.

When electric machines need to work with engines for HEVs, there are additional requirements:

- High-efficiency energy generation over wide-speed operation.

- Good voltage regulation over wide-speed generation.

- Capable of integrating with the engine.

\section{EV Machines for Electric Propulsion}

The classification of EV machines for electric propulsion is depicted in Fig. 1 where the bold and colored ones have been applied to EVs, including the PM DC, series DC, shunt DC, separately excited DC, cage-rotor induction, PM synchronous (PMSyn), PM brushless DC (PMBDC) and switched reluctance (SR) machines. Essentially, EV machines are classified as the commutator type which has a 
commutator and carbon brushes, and the commutatorless type which has no commutator. In recent years, the development trend is to develop different types of commutatorless machines [3], especially the class of doubly-salient machines and the class of vernier machines.

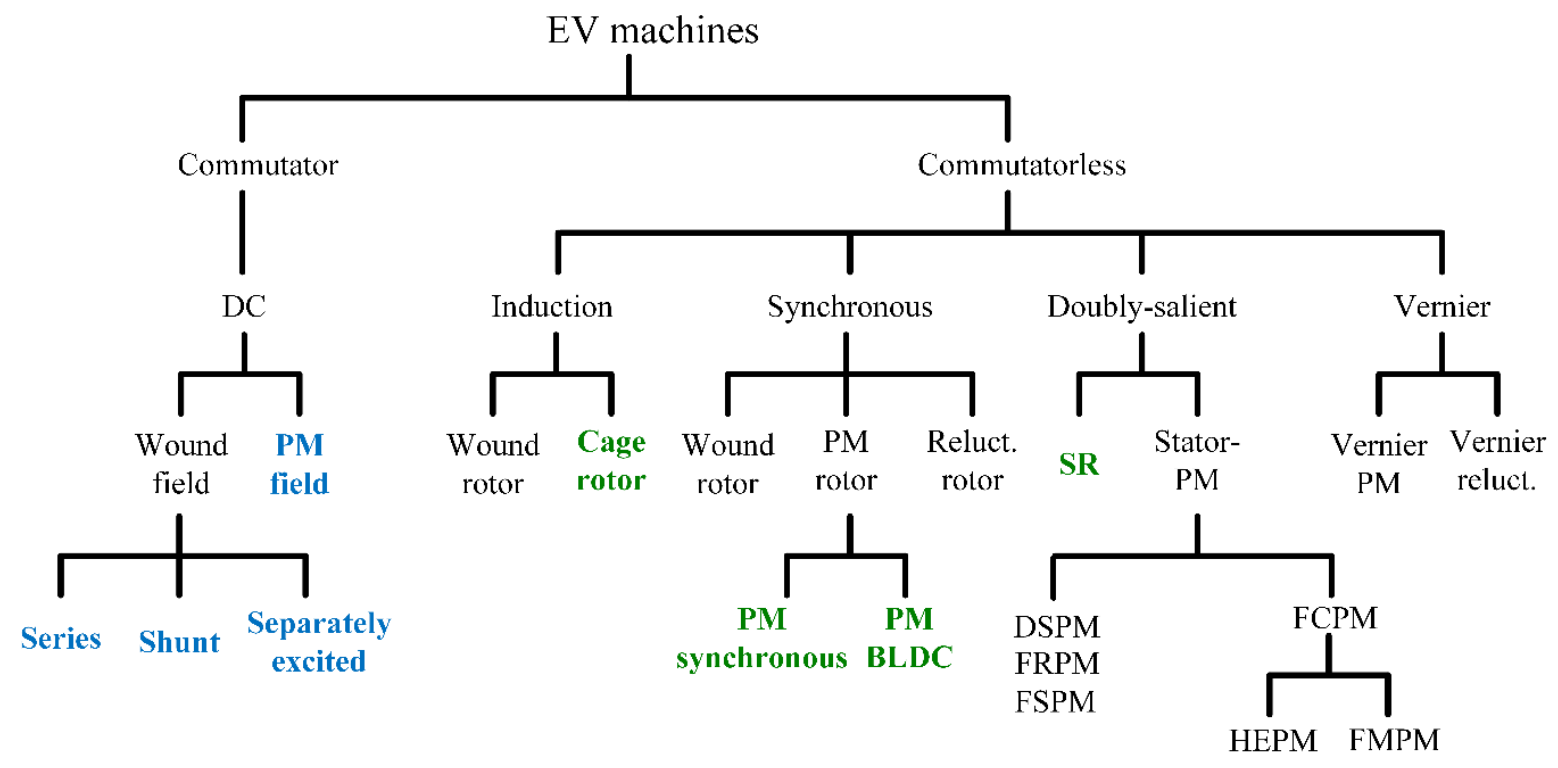

Fig. 1. Classification of EV machines for electric propulsion

There are many kinds of doubly-salient machines. Their key feature is the presence of salient poles in both the stator and rotor. The SR machine is the simplest kind of doubly-salient machines. When incorporating PMs into the stator of doubly-salient machines, the stator-PM machines are derived [4]. Since the rotor has no PMs or windings, they can retain high torque and power densities while offering mechanical simplicity and robustness, which are desirable for vehicular operation. Due to different locations of the PMs, these stator-PM machines can be specified as the doubly-salient PM (DSPM), flux-reversal PM (FRPM) and flux-switching PM (FSPM) machines. By adding DC field windings and magnetizing windings in the stator for flux control, they can be further specified as the hybrid-excited PM (HEPM) and flux-mnemonic PM (FMPM) versions, respectively, which are collectively termed the flux-controllable PM (FCPM) versions. When all PM poles are replaced with DC field windings, the doubly-salient DC (DSDC), flux-reversal DC (FRDC) and flux-switching DC (FSDC) machines are derived, which are advanced magnetless machines that can offer very low cost and flexible flux control.

By properly selecting the numbers of salient poles in the stator and rotor, the vernier machines can utilize the vernier effect to amplify the output torque while stepping down the speed, which is very desirable for low-speed high-torque direct-drive vehicles. They consist of the vernier PM (VPM) and vernier reluctance (VR) machines. The VPM machines can be rotor-PM or stator-PM, depending on the location of PMs. The rotor-PM VPM machine is most mature, and loosely termed the VPM machine [5], while the stator-PM VPM machine is also termed the vernier hybrid machine [6]. Without using PM excitation, the VR machines are structurally similar to the SR machine, but they operate differently. Because of their inherently low power factor, the VR machines can equip with an additional field winding, either DC or AC, in the stator to form the doubly-fed VR (DFVR) machine [7]. The VR and DFVR machines are also classified as advanced magnetless machines.

All EV machine topologies can readily be extended from the radial-flux morphology to other morphologies such as the axial-flux and transverse-flux morphologies [8]. The axial-flux morphology possesses the merits of higher torque and power densities than its radial-flux counterpart, but suffers from the drawback of axial force between the stator and rotor. The transverse-flux morphology can offer the highest torque density over its counterparts, but the corresponding machine structure is very complicated which hinders its practicability. 


\section{EV MAChINES FOR HYbRID PROPUlSION}

The classification of EV machine systems for hybrid propulsion is depicted in Fig. 2, where the bold and colored ones have been applied to HEVs including the integrated-starter-generator (ISG) system is for the micro HEV and mild HEV, and the ECVT system is for the full HEV and plug-in HEV (PHEV) [9]. The ISG system aims to offer not only the conventional features of engine cranking and electricity generation, but also the hybrid features of idle stop-start, regenerative braking and power assistance. Consequently, the machine designs are very demanding [10]. The ECVT system aims to offer continuously variable transmission from the engine to the wheels in an electronic way, hence achieving optimal fuel economy while retaining all hybrid features including the idle stop-start, regenerative braking and power assistance plus the unique feature of electric launch. Among three main types of ECVT systems: the planetary-geared ECVT (PG-ECVT), double-rotor ECVT (DR-ECVT) and magnetic-geared EVT (MG-ECVT), the PG-ECVT system is exclusively used for the buyable full HEV and PHEV [11]. However, this PG-ECVT system suffers from the fundamental drawback of planetary gearing such as the transmission loss, gear noise and lubrication requirement. In order to get rid of the planetary gear, the gearless DR-ECVT system has been proposed, which utilizes the double-rotor machine to replace the planetary gear for power split [12]. However, this DR-ECVT system suffers from the drawback of using slip-rings and carbon brushes to extract the energy from the inner rotor such as the reliability problem and maintenance requirement. By replacing the planetary gear with the magnetic planetary gear or magnetic coaxial gear, the MG-ECVT system can retain the merits of magnetic gearing and prevent from using slip-rings and carbon brushes so as to achieve pseudo-gearless and brushless [13]. However, this MG-ECVT system is so complicated that it demands precision manufacturing.

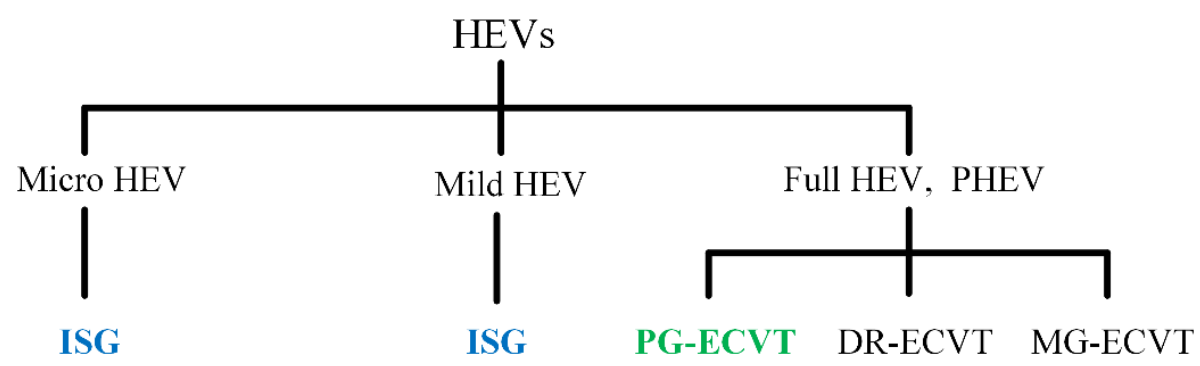

Fig. 2. Classification of EV machine systems for hybrid propulsion

\section{Challenges AND OPPORTUNities}

The stator-PM machines have high opportunities for EVs because they can solve two fundamental problems of those PM brushless brushless machines, including the PMSyn and PMBDC machines. Namely, there are no PMs in the rotor, which can avoid the problem on mounting PMs on the highspeed rotor and hence improving the mechanical integrity; and all PMs are located in the stator, which can enhance the cooling arrangement and hence improving the thermal stability of PMs. Those major types of stator-PM machines for electric propulsion, namely the FSPM, FRPM, DSPM and FCPM, are qualitatively compared as summarized in Table 1 . Since the FCPM machines are the flux-controllable versions of the three basic stator-PM machines, they consist of many members such as the HE-FSPM, FM-FSPM, HE-FRPM, FM-FRPM, HE-DSPM and FM-DSPM.

Concerning the torque and power densities, the FSPM machine is most preferable because of the flux-focusing arrangement. Meanwhile, the power density of the FCPM machines is degraded due to the use of additional DC field winding or magnetizing winding. Nevertheless, the developed torque, especially for electric launch or overtaking of EVs, can be temporarily boosted by instantaneous air-gap flux strengthening. Since the FCPM machines have the definite merit of flexible air-gap flux control, they can achieve higher efficiency than that of the basic FSPM, FRPM and DSPM machines. Concerning controllability, the FCPM machines are much better than those singly-fed stator-PM machines. As the PM material is relatively delicate, the PM immunity to accidental demagnetization and mechanical abuse are a key performance index for PM machines. Among those stator-PM machines, the FRPM machine has a relatively weak PM immunity because their PMs are mounted on the surfaces of stator poles so that they are prone to be partially demagnetized under high armature field or high temperature operation, 
and vulnerable to be physical damaged under severe vibration. Concerning robustness and manufacturability, the DSPM machine is most preferable because its structure is simplest in both the stator and rotor, leading to be highly robust and easily manufacturing. In contrast, the FCPM machines have to install two sets of windings (armature and field windings) in the stator, which inevitably degrades their manufacturability. Concerning maturity, the DSPM machine is most mature as it has been developed for over two decades. Then, the FSPM and FRPM machines are becoming mature, which have been developed for over a decade. The FCPM machines are recently derived from the singly-fed stator-PM machines so that they are relatively immature.

The VPM machines have the definite advantage of low-speed high-torque operation, which is essential for in-wheel direct-drive application. When adopting the conventional inner-rotor structure, the VPM machines need a mechanism to transmit the inner-rotor torque to the wheel, which definitely degrades their practicability. On the contrary, when adopting the outer-rotor structure, the VPM machines do not need any mechanism to transmit the outer-rotor torque to the wheel as the outer rotor is directly coupled with the wheel rim. Moreover, the inner stator of the outer-rotor structure can be fully utilized to accommodate the armature winding and the optional DC field winding. Because of the fact that most EV manufacturers do not prefer to use the in-wheel direct drive, the development of VPM machines for electric propulsion is much slower than expected. With ever increasing demand on higher performance and better stability, it is anticipated that the VPM machines will be popular for in-wheel direct-drive EVs.

Table 1. Evaluation of stator-PM motor drives for EVs

\begin{tabular}{lllll}
\hline & FSPM & FRPM & DSPM & FCPM \\
\hline Torque density & High & Good & Medium & High \\
Power density & High & Good & Medium & Medium \\
Efficiency & Good & Good & Good & High \\
Controllability & Medium & Medium & Medium & Superb \\
PM immunity & Good & Weak & Medium & Good \\
Robustness & Medium & Medium & High & Medium \\
Manufacturability & Medium & Medium & Easy & Hard \\
Maturity & Medium & Medium & High & Low \\
\hline
\end{tabular}

Because of the high price and fluctuating supply of rare-earth elements for PM materials, the advanced magnetless machines will be the core for EVs desiring high cost-effectiveness. The major types of advanced magnetless machines that are viable for electric propulsion are the DSDC, FSDC, VR, DFVR, and axial-flux magnetless (AFM) are compared as summarized in Table 2. It should be that the AFM machines refer to the axial-flux versions of those viable radial-flux magnetless machines such as the DSDC and FSDC machines.

Table 2. Comparison of potentiality of advanced magnetless motor drives

\begin{tabular}{llllll}
\hline & FSDC & DSDC & VR & DFVR & AFM \\
\hline Torque density & High & Good & High & High & Superb \\
Power density & High & Good & Low & Fair & Superb \\
Efficiency & High & High & Fair & High & High \\
Controllability & High & High & Fair & High & High \\
Power factor & Fair & Fair & Low & Fair & Fair \\
Material cost & Fair & Fair & Low & Fair & Fair \\
Robustness & Fair & Fair & High & Fair & Low \\
Manufacturability & Fair & Fair & Easy & Fair & Hard \\
Maturity & Fair & Fair & High & Low & Low \\
\hline
\end{tabular}

Concerning the torque and power densities, the AFM machines are most preferable as they can fully utilize the material for torque production. Since the FSDC machine exhibits bipolar flux linkage, it offers higher torque and power densities than the DSDC machine. While both the VR and DFVR machines have the vernier effect, they can offer high torque density for low-speed operation. Concerning the efficiency, controllability and power factor, the doubly-fed magnetless machines having the capability 
of field control, namely the FSDC, DSDC, DFVR and AFM, can achieve better performances than the singly-fed VR machine. On the contrary, concerning the material cost, robustness, manufacturability and maturity, the VR machine can offer better performances than those doubly-fed magnetless machines. In particular, the AFM machines desire the double-stator or double-rotor structure, which significantly deteriorates their robustness, manufacturability and maturity. Meanwhile, the FSDC and DSDC machines are considered as the most prominent magnetless alternatives. Both the DFVR and AFM machines are relatively most immature.

In the foreseeable future, it is anticipated that both EVs and HEVs will coexist in the market. Among all kinds of HEVs, the full HEV and PHEV are most attractive. As the ECVT is their core technology, the MG-ECVT system exhibits high opportunities for future HEVs. Compared with other ECVT systems, the MG-ECVT system has the definite advantages of pseudo-gearless and brushless, which can significantly improve the system efficiency, power density and operability for hybrid propulsion. The key challenges of this system are mainly the high initial cost due to the utilization of much PM materials and the complex structure which desires precision manufacturing. Being fueled by continual development of new PM materials and high-precision manufacturing techniques, this MG-ECVT system is highly promising for hybrid propulsion in near future.

\section{ACKNOWLEDGMENT}

This work was supported by a grant (Project No. 17200614) from the Hong Kong Research Grants Council, Hong Kong Special Administrative Region, China.

\section{REFERENCES}

[1] Z.Q. Zhu and D. Howe, "Electrical machines and drives for electric, hybrid and fuel cell vehicles," Proceedings of IEEE, vol. 95, 2007, pp. 746-765.

[2] K.T. Chau and W. Li, "Overview of electric machines for electric and hybrid vehicles," International Journal of Vehicle Design, vol. 64, 2014, pp. 46-71.

[3] K.T. Chau, C.C. Chan, and C. Liu, "Overview of permanent-magnet brushless drives for electric and hybrid electric vehicles," IEEE Transactions on Industrial Electronics, vol. 55, 2008, pp. 22462257.

[4] C. Liu, K.T. Chau, J.Z. Jiang, and S. Niu, "Comparison of stator-permanent-magnet brushless machines," IEEE Transactions on Magnetics, vol. 44, 2008, pp. 4405-4408.

[5] J. Li, K.T. Chau, and W. Li, "Harmonic analysis and comparison of permanent magnet vernier and magnetic-geared machines," IEEE Transactions on Magnetics, vol. 47, 2011, pp. 3649-3652.

[6] E. Spooner and L. Haydock, "Vernier hybrid machines," IEE Proceedings - Electric Power Applications, vol. 150, 2003, pp. 655-662.

[7] S. Taibi, A. Tounzi, and F. Piriou, "Study of a stator current excited vernier reluctance machine," IEEE Transactions on Energy Conversion, vol. 21, 2006, pp. 823-831.

[8] K.T. Chau, Electric Vehicle Machines and Drives - Design, Analysis and Application. Wiley-IEEE Press, 2015.

[9] K.T. Chau and C.C. Chan, "Emerging energy-efficient technologies for hybrid electric vehicles," Proceedings of IEEE, vol. 95, 2007, pp. 821-835.

[10] C. Liu, K.T. Chau, and J.Z. Jiang, "A permanent-magnet hybrid brushless integrated startergenerator for hybrid electric vehicles," IEEE Transactions on Industrial Electronics, vol. 57, 2010, pp. 4055-4064.

[11] M. Kamiya, "Development of traction drive motors for the Toyota hybrid system," IEEJ Transactions on Industry Applications, vol. 126, 2006, pp. 473-479.

[12] M. Hoeijmakers and J. Ferreira, "The electric variable transmission," IEEE Transactions on Industry Applications, vol. 42, 2006, pp. 1092-1100.

[13] L. Jian and K.T. Chau, "A novel electronic-continuously variable transmission propulsion system using coaxial magnetic gearing for hybrid electric vehicles," Journal of Asian Electric Vehicles, vol. 7, 2009, pp. 1291-1296. 